\title{
OMENTAL LEIOMYOSARCOMA WITH UNUSUAL GIANT CELLS IN A BEAGLE DOG - SHORT COMMUNICATION
}

\author{
Jun SASAKI $^{1 *}$, Megumi ToYOSHIMA ${ }^{1}$, Yasuhiko OKAMURA ${ }^{2}$ and Masanobu GORYO ${ }^{1}$ \\ ${ }^{1}$ Department of Veterinary Pathology, ${ }^{2}$ Veterinary Surgery, Faculty of Agriculture, \\ Iwate University, 3-18-8 Ueda, Morioka, Iwate 020-8550, Japan
}

(Received 26 May 2015; accepted 28 October 2015)

\begin{abstract}
A 10-year-old castrated male Beagle dog was presented with a 2-month history of intermittent vomiting and abdominal pain. The dog was referred to the Veterinary Teaching Hospital at Iwate University for further evaluation, and a splenic tumour was suspected on the basis of ultrasonography and computed tomography. Surgery identified a large, solid, light-pink mass on the greater omentum with blood-coloured ascites in the abdominal cavity, and resection was performed. Microscopically, the mass comprised spindle-shaped tumour cells and scattered osteoclast-like giant cells. Most spindle-shaped cells were positive for vimentin, desmin, and smooth muscle actin ( $\alpha$-SMA), whereas osteoclast-like giant cells were positive only for vimentin. On the basis of histopathological and immunohistochemical findings, a diagnosis of leiomyosarcoma was made. To the best of our knowledge, this represents the first report of leiomyosarcoma associated with osteoclast-like giant cells developing from the greater omentum in a dog.
\end{abstract}

cell

Key words: Dog, leiomyosarcoma, greater omentum, osteoclast-like giant

Leiomyosarcoma commonly occurs in the gastrointestinal and female genital tracts in domestic animals (Hendrick et al., 1998). This malignant tumour of soft tissue is composed of spindle-shaped cells showing cigar-shaped nuclei. Leiomyosarcoma containing osteoclast-like giant cells has been reported in human cases (Matthews and Fisher, 1994; Sarma et al., 2007; Gibbons et al., 2010), but does not appear to have been previously described in domestic animals. The present report documents the histopathological, immunohistochemical and electron microscopic features of omental leiomyosarcoma with osteoclast-like giant cells in a dog.

A 10-year-old castrated male Beagle dog developed intermittent vomiting and abdominal pain and was referred to the Veterinary Teaching Hospital at Iwate University for further evaluation. A splenic tumour was suspected on the basis of ultrasonography and computed tomography (CT) findings, as the spleen was seen to have been displaced to behind the liver by a large abdominal mass

*Corresponding author; E-mail: sasajun@iwate-u.ac.jp; Phone: 0081 (19) 621-6166 
(Fig. 1). The possibility of continuity between the large abdominal mass and the spleen was unclear on CT. The large abdominal mass was solid, firmly elastic, and light pink in colour. The mass was found to be occupying the entire abdominal cavity, and surgical resection was therefore performed. The large abdominal mass measured $15.6 \times 10.2 \times 7.0 \mathrm{~cm}$ in size, and weighed $584 \mathrm{~g}$ (Fig. 2). The cut surface of the mass was creamy-white and solid. No mass or nodular lesions were evident on the spleen, although adherence to the large abdominal mass was confirmed. No tumour masses of similar appearance were observed in any other organs. Large amounts of blood-coloured ascites were evident in the abdominal cavity. The dog died three months after surgery, and unfortunately it was not possible to perform an autopsy.

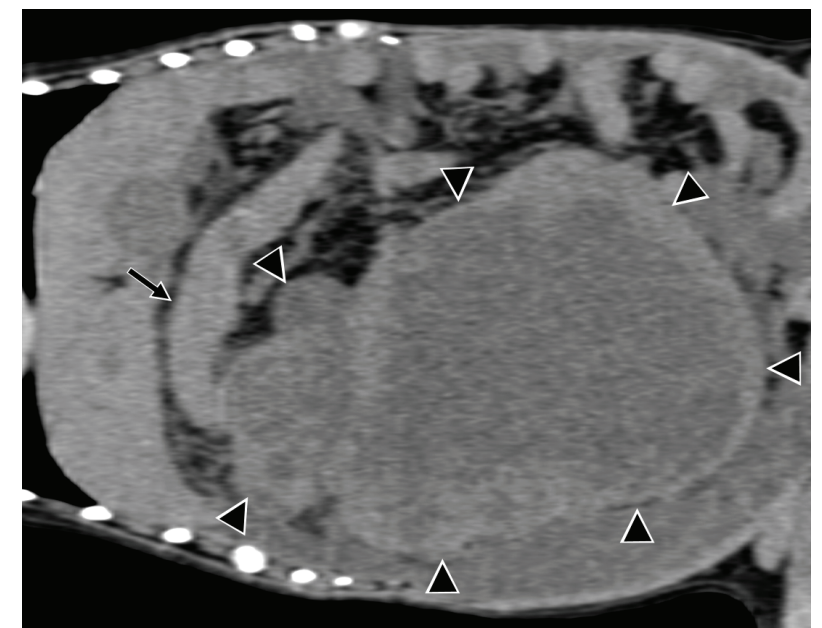

Fig. 1. Coronal-section abdominal CT, showing a large abdominal mass (arrowheads). The spleen has been displaced to behind the liver (arrow)

The resected mass was examined histopathologically. Samples were fixed in $10 \%$ formalin and embedded in paraffin wax. Sections $(4 \mu \mathrm{m})$ were stained with haematoxylin and eosin (HE). The mass comprised numerous spindleshaped tumour cells with elongated or ovoid nuclei, and cigar-shaped nuclei were sometimes observed (Fig. 3A). Abundant eosinophilic cytoplasm was present, and the tumour cells formed broad, interlacing fascicles. Osteoclast-like multinucleated giant cells were scattered throughout the mass (Fig. 3B). These showed a more eosinophilic cytoplasm and multiple nuclei. Spindle-shaped tumour cells showed many mitotic figures, but the osteoclast-like giant cells showed no mitosis. Multifocal haemorrhagic areas and areas of widespread necrosis were also observed within the mass. Silver impregnation for reticulin fibres revealed thin reticular fibres around the individual tumour cells. Masson's trichrome staining showed a small amount of collagen fibres in the stroma. No 
positive findings for intracytoplasmic lipid were obtained using Sudan III staining. No spindle-shaped tumour cells or proliferation of multinucleated giant cells were seen in the spleen, although invasive proliferation of tumour cells was observed at the spleen in the area attached to the mass.

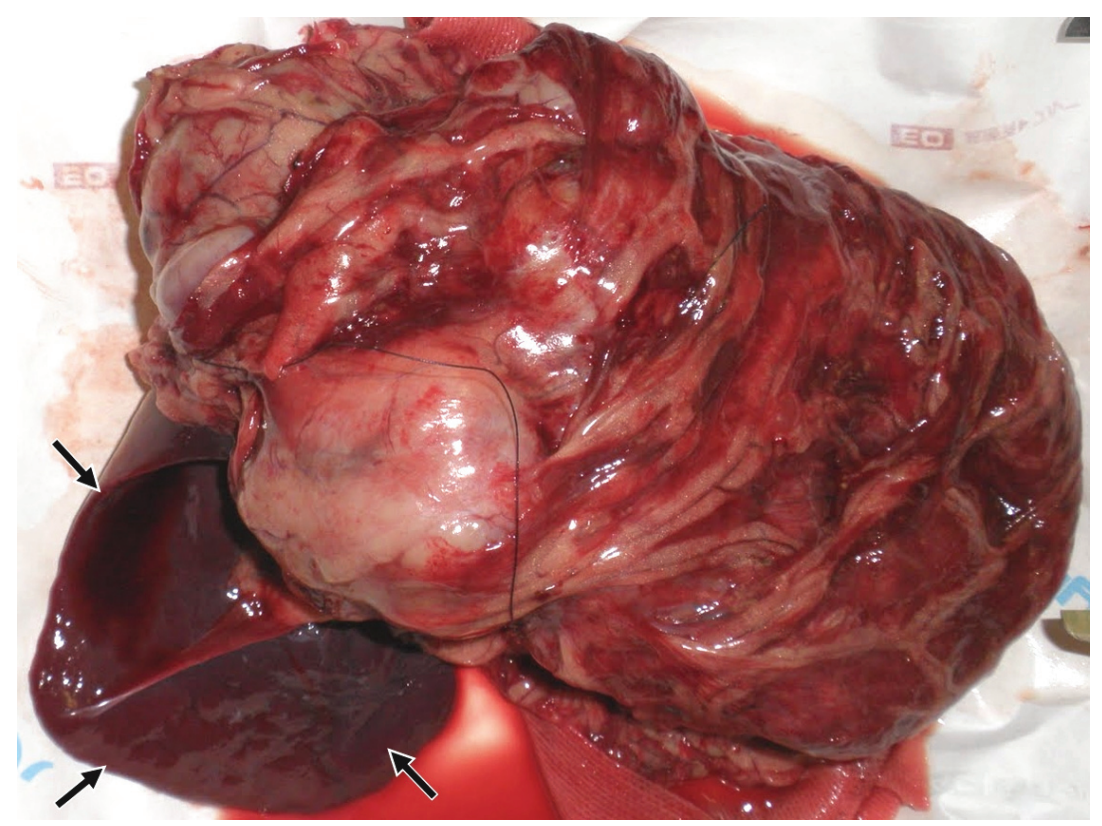

Fig. 2. Large mass with blood-red coloration in the peritoneum. No nodular lesions are apparent in the spleen (arrows)

Immunohistochemical staining using primary antibodies was performed on 4- $\mu \mathrm{m}$ sections of the mass (Table 1). For antigen retrieval, sections stained for vimentin, desmin, $\alpha$-SMA, myoglobin, S100 protein, factor-VIII, cytokeratin (Pan), HLA-DR, myeloid/histiocyte antigen, Iba1, $\alpha 1$-antitrypsin, CD68, CD117, TRAP, and Ki-67 were heated at $121^{\circ} \mathrm{C}$ for $15 \mathrm{~min}$ in a 10 -mM sodium acid citrate-buffered solution. Antigen retrieval for lysozyme was performed with $0.1 \%$ trypsin at $37^{\circ} \mathrm{C}$ for $30 \mathrm{~min}$. The labelled streptavidin-peroxidase-complex method was performed according to the manufacturer's (LSAB ${ }^{\circledR}+$ System-HRP, Dako) instructions, followed by application of 3,3'-diaminobenzidine. Sections were counterstained with Mayer's haematoxylin. Canine intestine and lymph node were used as positive control. Immunohistochemical analysis showed that most spindle-shaped tumour cells were strongly positive for vimentin, desmin (Fig. 3C) and $\alpha$-SMA. These spindle-shaped tumour cells showed negative results for myoglobin, S100 protein, factor-VIII, cytokeratin (Pan), HLA-DR, myeloid/ histiocyte antigen, lysozyme, Iba1, $\alpha 1$-antitrypsin, CD68, CD117, and TRAP. 
Osteoclast-like giant cells were positive only for vimentin. The Ki-67 labelling index was calculated from the number of Ki-67-positive cells among a total of $1,000$ cells (from 10 high-powered [ $\times 400]$ fields), as approximately $39.8 \%$ (Fig. 3D).

Electron microscopic examination of a formalin-fixed sample revealed that spindle-shaped tumour cells had cytoplasmic filaments with dense bodies, rough endoplasmic reticulum, mitochondria, and dense patches in association with the cytoplasmic membrane. Multinucleated giant cells showed large amounts of rough endoplasmic reticulum, but other cell organelles were not obvious.

The present case showed typical histopathological, immunohistochemical and electron microscopic features of leiomyosarcoma, with the exception of the appearance of the numerous osteoclast-like multinucleated giant cells. Leiomyosarcoma associated with osteoclast-like giant cells has been reported in humans (Matthews and Fisher, 1994; Sarma et al., 2007; Gibbons et al., 2010), but similar cases do not appear to have been reported in domestic animals. The osteoclastlike giant cells in leiomyosarcoma of the human skin were strongly positive for CD68, indicating that the osteoclast-like giant cells represented reactive histiocytic cells (Sarma et al., 2007). Another report indicated that osteoclast-like giant cells were formed from tumour-associated macrophages (Gibbons et al., 2010). In the present case, most spindle-shaped tumour cells were positive for vimentin, desmin and $\alpha$-SMA, although the osteoclast-like giant cells were only positive for vimentin. Further studies are needed to clarify the role of osteoclast-like giant cells in leiomyosarcoma. Among domestic animals, only one case of bovine leiomyosarcoma containing atypical giant cells has been reported (Hanzaike et al., 1995). Those giant cells showed positive staining with antibody against $\alpha$ SMA, suggesting cellular pleomorphism of leiomyosarcoma in that case.

Although leiomyosarcoma can arise as a primary splenic neoplasm (Spangler et al., 1994), no mass or nodules were present in the spleen in this case. Moreover, no tumour cells were seen in any other areas of the spleen, although histopathological examination revealed mild adhesion and slight invasive proliferation of spindle tumour cells between the splenic capsule and the abdominal mass. According to our examination, the greater omentum was considered highly likely to represent the original site of leiomyosarcoma.

The leading differential diagnosis to leiomyosarcoma in the greater omentum is gastrointestinal stromal tumour (GIST). GIST has been reported in dogs and other animals, and has been known to typically express KIT (CD117) (Maas et al., 2007). In the present study, the neoplastic spindle cells and osteoclast-like giant cells did not show the features of GIST, such as CD117-positive immunoreactivity.

On the basis of the histopathological, immunohistochemical and electron microscopic findings, a diagnosis of leiomyosarcoma was made. To the best of our knowledge, this represents the first report of leiomyosarcoma with osteoclast-like giant cells in a dog. 


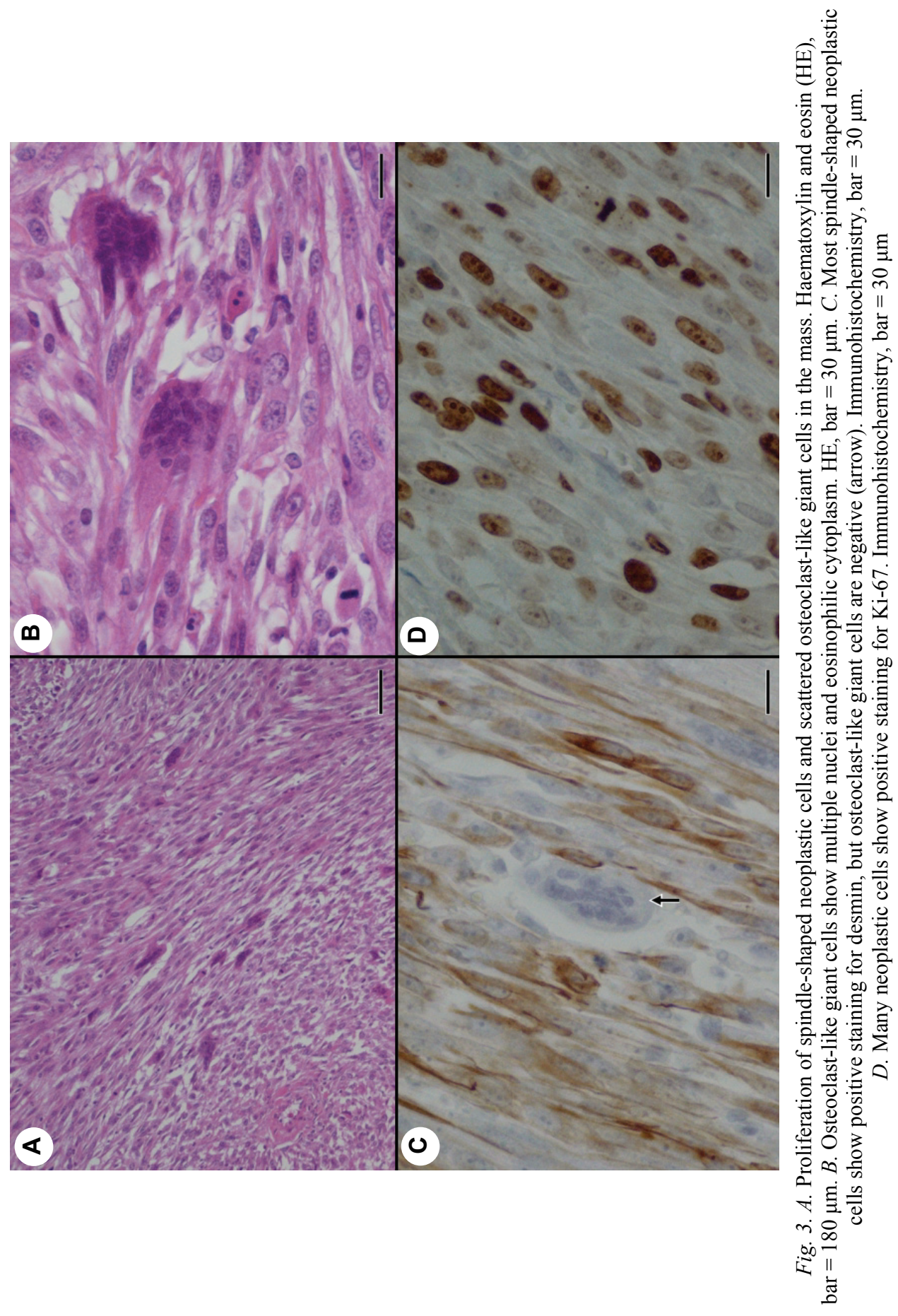


OMENTAL LEIOMYOSARCOMA IN A DOG

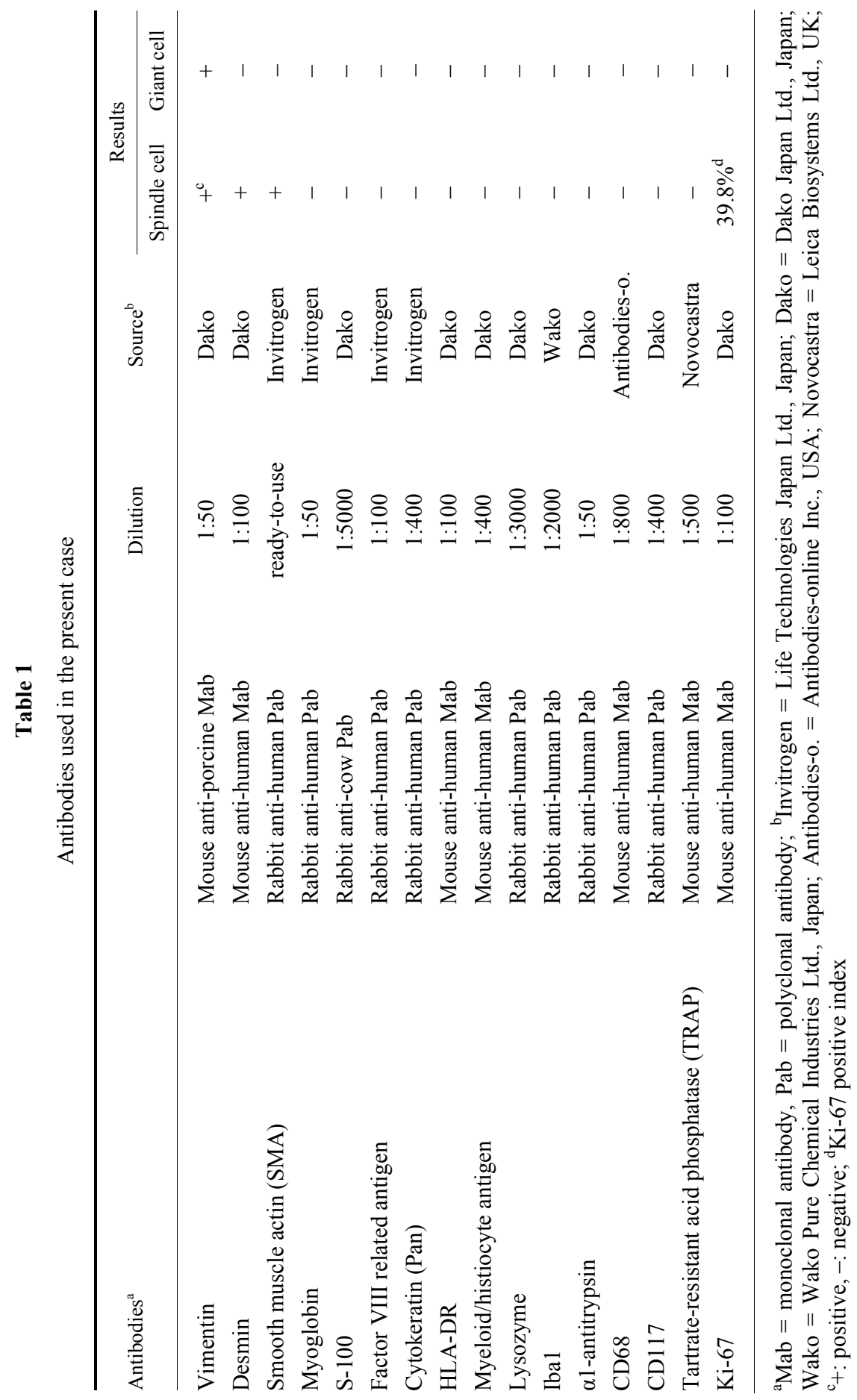




\section{References}

Gibbons, C. L., Sun, S. G., Vlychou, M., Kliskey, K., Lau, Y. S., Sabokbar, A. and Athanasou, A. (2010): Osteoclast-like cells in soft tissue leiomyosarcomas. Virchows Arch. 456, 317-323.

Hanzaike, T., Ito, I., Ishikawa, T., Ishikawa, Y. and Kadota, K. (1995): Leiomyosarcoma of soft tissue in a cow. J. Comp. Path. 112, 237-242.

Hendrick, M. J., Mahaffey, E. A., Moore, F. M., Vos, J. H. and Walder, E. J. (1998): Tumors of smooth muscle. In: Histological Classification of Mesenchymal Tumors of Skin and Soft Tissues of Domestic Animals. Second Series Volume II. World Health Organization, Washington, D.C. pp. 20-21.

Maas, C. P., ter Haar, G., van der Gaag, I. and Kirpensteijn, J. (2007): Reclassification of small intestinal and cecal smooth muscle tumors in 72 dogs: clinical, histologic, and immunohistochemical evaluation. Vet. Surg. 36, 302-313.

Matthews, T. J. and Fisher, C. (1994): Leiomyosarcoma of soft tissue and pulmonary metastasis, both with osteoclast-like giant cells. J. Clin. Pathol. 47, 370-371.

Sarma, D. P., Santos, E. E. and Wang, B. (2007): Leiomyosarcoma of the skin with osteoclast-like giant cells: a case report. J. Med. Case Rep. 1, 180.

Spangler, W. L., Culbertson, M. R. and Kass, P. H. (1994): Primary mesenchymal (nonangiomatous/nonlymphomatous) neoplasms occurring in the canine spleen: Anatomic classification, immunohistochemistry, and mitotic activity correlated with patient survival. Vet. Pathol. 31, 37-47. 